\title{
RISCOS OCUPACIONAIS IDENTIFICADOS NOS AMBIENTES DE PANIFICAÇÃO BRASILEIROS ${ }^{1}$
}

\author{
OCCUPATIONAL RISKS IDENTIFIED IN BRAZILIAN \\ BAKERY ENVIRONMENTS
}

\section{RIESGOS OCUPACIONALES IDENTIFICADOS EN LOS AMBIENTES DE PANIFICACIÓN BRASILEIROS}

\author{
Maria Emilia de Paula Denipotti * \\ Maria Lúcia do Carmo Cruz Robazzi ${ }^{* *}$
}

\begin{abstract}
RESUMO
Estudo exploratório descritivo de abordagem quantitativa, com coleta de dados realizado em 2008, que objetivou caracterizar o perfil pessoal e profissional e identificar os riscos ocupacionais (RO) vivenciados por trabalhadores da panificação de 14 padarias em uma cidade interiorana do Estado de São Paulo, Brasil. Os dados foram obtidos por meio de um questionário sobre a identificação pessoal e profissional de trabalhadores e fatores de RO. De 47 entrevistados, a maioria é homem, casada, com ensino médio incompleto, idade entre 31 a 40 anos, atua de 10 a 15 anos na panificação, não apresenta emprego anterior e trabalha 8 horas diárias. Os fatores de RO identificados foram: calor, ruído, poeira de farinha de trigo, levantamento de peso, movimentos repetitivos, presença de gripes e resfriados, entre outros; a maioria, ao final da jornada de trabalho, manifesta fadiga física. Conclui-se que os sujeitos conseguem identificar alguns dos fatores de risco a que estão expostos. Ações de orientação à saúde podem ser desenvolvidas visando à minimização dos RO.
\end{abstract}

Palavras chave: Trabalhadores, riscos ocupacionais, saúde do trabalhador.

\begin{abstract}
This exploratory, descriptive and quantitative study aimed to characterize the personal and occupational profile and to identify the Occupational Risks (OR) experienced by baking workers from 14 bakeries in a city in the interior of the state of São Paulo, Brazil. Data were collected, in 2008, using a questionnaire about workers' personal and professional identification and OR factors. From 47 subjects, most are men, married, did not complete secondary education, aged between 31 and 40 years, have worked from 10 to 15 years in bakery, did not have previous job and worked 8 hours per day. OR factors identified were: heat, noise, wheat flour dust, weight lifting, repetitive movements, flu and colds, among others. Most workers report physical fatigue at the end of the workday. It is concluded that workers are able to identify some risk factors to which they are exposed. Health guidance actions can be developed aiming to minimize the OR.
\end{abstract}

Key words: Workers, occupational risks, occupational health.

\footnotetext{
${ }^{1}$ Este artigo foi extraído da monografia intitulada "Riscos Ocupacionais e Agravos à Saúde em trabalhadores da panificação no município de Orlândia/SP", a qual foi requisito para a finalização do Curso de Especialização em Enfermagem do Trabalho pela Escola de Enfermagem de Ribeirão Preto da Universidade de São Paulo (USP), Ribeirão Preto, Brasil.

* Enfermeira, Escola de Enfermagem de Ribeirão Preto da Universidade de São Paulo, Ribeirão Preto, Brasil. Email: mepdenipotti@yahoo.com.br.

** Enfermeira. Professora do Departamento de Enfermagem Universidade de São Paulo (USP), Ribeirão Preto, São Paulo, Brasil.Email: avrmlcc@eerp.usp.br.
} 


\section{RESUMEN}

Estudio exploratorio, descriptivo y cuantitativo que tuvo como objetivo caracterizar el perfil personal y laboral e identificar los Riesgos Laborales (RL) de los trabajadores de 14 panaderías en una ciudad en el interior del estado de São Paulo, Brasil. Los datos fueron recolectados, en el año 2008, a través de un cuestionario acerca de la identificación personal y profesional de los trabajadores y los factores de RL. De los 47 sujetos del estudio, la mayoría es hombre, casada, con enseñanza media incompleta, edad entre 31 y 40 años, actuaban hacía 10 a 15 años en la panificación, no tuvieron empleo anterior y trabajaban 8 horas diarias. Los factores de RL identificados fueron: calor, ruido, polvo de harina de trigo, levantamiento de peso, movimientos repetitivos, gripes y resfriados, entre otros. La mayoría menciona fatiga física al final de la jornada. Se concluye que los sujetos identifican los factores de RL a que están expuestos. Acciones de orientación en salud pueden ser desarrolladas permitiendo la minimización de los RL.

Palabras clave: Trabajadores, riesgos laborales, salud laboral.

Fecha recepción: 31/07/2019 Fecha aceptación: 12/04/2011

\section{INTRODUÇÃO}

O pão foi um dos primeiros alimentos preparados e transformados por mãos humanas e a panificação acompanha a evolução da humanidade. No Brasil, seu surgimento é por volta do Século XIX e com o avanço tecnológico nesta área, foram criadas máquinas para diminuir o custo e aumentar a produção. Entretanto, até hoje, parece não haver muita preocupação com as condições laborais e de saúde dos trabalhadores da panificação. A idéia de realizar o presente estudo surgiu após alguns atendimentos à saúde, realizados pelos autores, enfermeiros, aos trabalhadores responsáveis pela fabricação de pães, o que os levou a procurar conhecer melhor este processo de trabalho em padarias nacionais.

Quando o consumidor chega a uma padaria para comprar seus produtos percebe balcões e prateleiras bem organizados, caracterizando um ambiente convidativo e agradável. O processo de panificação, muitas vezes, ainda é artesanal, não se encontra visível e, conseqüentemente, não são constatados os diversos fatores de Riscos Ocupacionais (RO) presentes nos ambientes da fabricação de pães.

Risco indica a probabilidade de que um erro ocorra (1). Ambientes de trabalho po- dem apresentar diversificados agentes/fatores de riscos que se constituem nos RO. Além dos fatores nocivos ambientais, há as condições físicas, organizacionais, administrativas ou técnicas existentes nos locais de trabalho, que podem propiciar a ocorrência de acidentes de trabalho e/ou adoecimentos (2). Fatores químicos, físicos, mecânicos, biológicos e psicossociais de reconhecida ou suposta nocividade, presentes nos ambientes laborais são considerados RO (3).

A atividade laboral dos envolvidos no processo da panificação pode lhes propiciar conseqüências negativas para a saúde, sendo algumas delas as enfermidades alérgicas respiratórias.

Doenças ocupacionais alérgicas das vias respiratórias são comuns entre padeiros e a asma constitui-se em uma dessas manifestações (4). A exposição à poeira de farinha tem sido relatada como um importante fator de risco para tais doenças alérgicas (5).

Trabalhadores de padaria apresentam uma das maiores taxas de incidência de asma ocupacional, sendo 80 vezes mais propensos a desenvolver asma no trabalho do que o trabalhador médio britânico. A farinha contém diversas substâncias alergênicas; a fim de melhorar a qualidade e o processamento de massa de pão, esta farinha de panificação é suplementada com aditivos. 
Enzimas (por exemplo, fungos e bactérias alfa-amilase) são adicionadas para liberar o açúcar e fermentação do amido e essas e outras enzimas aumentam o risco de sensibilização e alergia entre os trabalhadores de panificação (6).

Elevada prevalência de sensibilização alérgica e asma foi identificada em padarias de supermercados da África do Sul, entre 211 indivíduos selecionados, aleatoriamente, em cinco categorias diferentes de trabalho, em 18 padarias. Ficou evidente que as estratégias de controle de exposição em padarias mostraram-se insuficientes para reduzir o contato com a poeira de farinha e proteger a saúde dos trabalhadores da panificação (5). A asma pode ser ocasionada por sensibilização imunológica aos alergênicos específicos e conseqüentes reações alérgicas de vias aéreas (4).

A relação entre a Doença Pulmonar Obstrutiva Crônica (DPOC), incluindo bronquite crônica e enfisema e a exposição à poeira de carvão está bem estabelecido; entretanto buscou-se rever as provas relativas a outras causas ocupacionais da DPOC. Evidenciou-se que a atopia tem papel importante no desenvolvimento da DPOC em trabalhadores de fábrica de farinha e de padarias, com os sujeitos sensibilizados às substâncias alergênicas, apresentando diminuição da função superior pulmonar, quando comparados aos não sensibilizados (7).

Além da asma, trabalhadores de panificação apresentam riscos de desenvolver outros sintomas respiratórios, como rinite. $\mathrm{Na}$ Suécia a exposição à poeira inalável de farinha foi medida em 12 padarias para se determinar as concentrações de poeira inalável no ar. Realizou-se o total de 129 medições, notando-se que há uma nítida hierarquia na média geométrica da exposição entre os trabalhadores, afetando mais os que manipulavam a massa de pão, seguidos dos que os montavam, dos que os colocavam nos fornos e, por último, dos embaladores e trabalhadores de confeitaria (8).

$\mathrm{Na}$ Holanda, investigação descreveu a as- sociação entre a exposição aos níveis de alergênicos de trigo e de sensibilização a estes alergênicos, relacionada com sintomas respiratórios superiores e inferiores e asma em trabalhadores de panificação (4).

Em relação aos riscos, sabe-se que vários fatores podem influenciar a sua percepção. Estudo objetivou fornecer uma visão de avaliação de risco na percepção dos trabalhadores na indústria de panificação, sendo 87 britânicos e 64 gregos. Importantes diferenças entre os países, na percepção de risco foram evidenciadas, por grupos específicos de trabalhadores como foi o caso dos que trabalham na indústria de panificação; falta uma melhor compreensão sobre como os trabalhadores caracterizam o risco (9).

$\mathrm{Na}$ Romênia, estudo objetivou identificar os riscos existentes em padarias e se tais riscos poderiam afetar a saúde dos trabalhadores por meio de indução de doenças profissionais ou de doenças relacionadas profissão, bem como poderiam agravar as doenças pré-existentes. Constatou-se que os trabalhadores poderiam desenvolver as seguintes doenças: rinite alérgica, asma brônquica, alveolite brônquica alérgica (pneumonia por hipersensibilização), síndrome reativa da via aérea, síndrome do túnel do carpo em decorrência de movimentos repetitivos, síndrome de compressão do nervo ciático por causa da posição viciosa do corpo, doença venosa crônica nos membros inferiores pela posição ortostática prolongada associada à manipulação de peso, choque calórico diante da exposição às temperaturas elevadas, entre outras (10).

$\mathrm{Na}$ literatura latino-americana ainda são escassas as investigações envolvendo os trabalhadores de panificação e os $\mathrm{RO}$ aos quais se submetem em seus ambientes de trabalho. Diante do exposto, visando aumentar o conhecimento sobre o tema, elaborou-se o presente estudo com o objetivo de caracterizar o perfil pessoal e ocupacional dos trabalhadores da panificação e identificar os RO presentes nos ambientes onde trabalham. 


\section{MATERIAL E MÉTODOS}

\section{Tipo e local do estudo}

Estudo não experimental, de caráter descritivo que utilizou análise quantitativa dos dados, realizado em um município brasileiro, do Estado de São Paulo (Brasil), cujos dados censitários indicam que a população, em 2008, era de 37.422 habitantes (9). O município tem 14 padarias.

\section{Período e instrumento de coleta de dados}

A coleta de dados realizou-se entre junho a agosto de 2008, por meio de utilização de um questionário elaborado pelos autores, baseado na literatura consultada.

Este instrumento continha 26 questões, a maioria de múltipla escolha e estava subdividido em três partes: 1 . Perfil do trabalhador (sexo, estado civil, escolaridade, idade, tempo de trabalho na panificação, atividade exercida no emprego anterior, horas de trabalho no emprego atual, outros vínculos empregatícios e carga horária nestes outros empregos), 2. Fatores de Riscos no Trabalho (físicos, químicos, ergonômicos, biológicos, psicossociais) e 3. Agravos à Saúde (acidentes de trabalho, adoecimentos, afastamentos por acidentes e doenças).

No presente estudo estão apresentados os resultados das partes 1 e 2 . O instrumento foi submetido à validação aparente e de conteúdo por especialistas em Saúde do Trabalhador, sofrendo ajustes. Após as alterações foi realizado um teste piloto com cinco padeiros de uma panificadora, na mesma cidade, para a verificação da sua compreensão sobre as questões. Os questionários respondidos durante este teste não foram incluídos nos resultados do estudo; os respondentes não demonstraram dificuldades em responder às questões e ao serem questionados sobre o que acharam das mesmas, relataram ter sido de fácil entendimento e adequado.
O teste mostrou, então, que o instrumento era factível. Além deste instrumento para coletar os dados, foram realizadas visitas para observação nos ambientes ocupacionais das padarias.

\section{Sujeitos}

As 14 padarias do município contavam com 147 empregados, entre balconistas, entregadores, pessoal de limpeza, confeiteiros, salgadeiros e, entre eles, 52 trabalhadores envolvidos, diretamente, no processo de produção da massa de pães.

Foram estabelecidos os seguintes critérios para a constituição dos sujeitos: - de inclusão: ser padeiro (participar do processo de produção da massa de pães e confecção dos mesmos), estar contratado nas padarias privadas ou pública, de ambos os sexos e concordar em participar da pesquisa, de modo voluntário. Também foram incluídos aqueles em período de férias, que estavam de licença ou demais tipos de afastamentos; - de exclusão: os que não consentiram participar do estudo e os que não participam do processo de produção de massa e confecção de pães (confeiteiros, auxiliares de limpeza, balconistas, entregadores, salgadeiros e outros). Obedecidos tais critérios, tornaram-se então sujeitos do estudo 47 padeiros (90,38\% em relação aos 52 envolvidos no processo de produção da massa).

\section{Procedimentos metodológicos}

Foram entregues as solicitações de autorizações para a realização do estudo aos 14 responsáveis pelas padarias, obtendo-se autorizações de todos.

Para o início da coleta de dados, estabeleceu-se contato telefônico com os proprietários/responsáveis e agendadas visitas para prestar esclarecimentos sobre o estudo aos trabalhadores. Durante as visitas foram conhecidas as instalações das padarias, sendo anotados os nomes e endereços residenciais 
dos padeiros, para posterior aplicação dos questionários nos domicílios. Após, foram agendados dia e hora com cada um, a fim de ser concretizada aplicação dos questionários.

A coleta de dados iniciou-se durante as visitas domiciliares, sendo posteriormente realizadas visitas nas padarias durante e após o horário de expediente; o que possibilitou a confirmação de várias das informações dos sujeitos e a ampliação do conhecimento sobre a realidade do seu trabalho. Os dados coletados foram inseridos em planilha de Excel, organizados em tabelas, analisados através de estatística descritiva e interpretados com embasamento no referencial teórico deste estudo.

\section{Procedimentos éticos}

Atendendo às recomendações da Resolução 196/96 do Conselho Nacional de Saúde brasileiro (11), o projeto foi encaminhado a um Comitê de Ética em Pesquisa (CEP), de uma
Instituição de Ensino Superior brasileira para análise, tendo sido considerado aprovado. Cada participante da pesquisa foi informado sobre o Termo de Consentimento Livre e Esclarecido; uma cópia deste documento foi entregue aos trabalhadores e a outra permanece com os pesquisadores.

\section{RESULTADOS}

A maioria dos 47 trabalhadores é do sexo masculino $(95,74 \%)$; houve o predomínio de casados $(55,32 \%)$, dos que possuem ensino médio incompleto $(34,04 \%)$ e entre 31 a 40 anos de idade. A caracterização dos seus dados profissionais, encontra-se apresentada na Tabela 1.

Os fatores/agentes de RO identificados pelos 47 sujeitos encontram-se apresentados a seguir.

Tabela 1. Distribuição de 47 trabalhadores da panificação segundo suas características profissionais. Brasil, 2008.

\begin{tabular}{|c|c|c|c|c|c|}
\hline Características profissionais & f & $\%$ & Características profissionais & $\mathbf{f}$ & $\%$ \\
\hline Tempo de trabalho na panificação (em anos) & \multicolumn{2}{|c|}{$(n=47)$} & Função no emprego anterior & \multicolumn{2}{|c|}{$(n=26)$} \\
\hline De 0 a 5 & 13 & 27.65 & Confeiteiro & 2 & 7.69 \\
\hline De 5 a 10 & 7 & 14.90 & S ervente de pedreiro & 3 & 11.54 \\
\hline De 10 a 15 & 16 & 34.04 & Pedreiro & 3 & 11.54 \\
\hline De 15 a 20 & 7 & 14.90 & Balconis ta de loja & 3 & 11.54 \\
\hline Mais de 20 & 4 & 8.51 & Guarda noturno & 3 & 11.54 \\
\hline Total & 47 & 100.00 & Operador de caixa s upermercado & 2 & 7.69 \\
\hline Carga horária diária/a tual emprego (em horas) & \multicolumn{2}{|c|}{$(n=47)$} & Chapeiro & 1 & 3.85 \\
\hline $07: 30$ & 1 & 2.12 & Trabalhador rural & 2 & 7.69 \\
\hline 08:00 & 36 & 76.60 & S ervente es colar & 1 & 3.85 \\
\hline 09:00 & 2 & 4.26 & Açougueiro & 1 & 3.85 \\
\hline $10: 00$ & 4 & 8.51 & Garçom & 2 & 7.69 \\
\hline $12: 00$ & 3 & 6.39 & Pajem & 1 & 3.85 \\
\hline $18: 00$ & 1 & 2.12 & Guarda & 2 & 7.69 \\
\hline Total & 47 & 100.00 & Total & 26 & 100.00 \\
\hline Emprego anterior & \multicolumn{2}{|c|}{$(n=47)$} & Função em outros empregos a tuais & \multicolumn{2}{|c|}{$(n=14)$} \\
\hline S im & 26 & 55.34 & Confeiteiro & 5 & 35.71 \\
\hline Não & 21 & 44.66 & Padeiro & 6 & 42.87 \\
\hline \multirow[t]{9}{*}{ Total } & 47 & 100.00 & Garçom & 1 & 7.14 \\
\hline & & & Mecânico & 1 & 7.14 \\
\hline & & & Vendedora & 1 & 7.14 \\
\hline & & & Total & 14 & 100.00 \\
\hline & & & Carga horária diária/outro emprego a tual & \multicolumn{2}{|c|}{$(n=14)$} \\
\hline & & & $4: 00 \mathrm{~h}$ & 12 & 85.72 \\
\hline & & & $3: 00 \mathrm{~h}$ & 1 & 7.14 \\
\hline & & & $6: 00 \mathrm{~h}$ & 1 & 7.14 \\
\hline & & & Total & 14 & 100.00 \\
\hline
\end{tabular}


Tabela 2. Distribuição de 47 trabalhadores da panificação quanto aos fatores de riscos ocupacionais que identificaram. Brasil, 2008.

\begin{tabular}{|c|c|c|c|c|c|}
\hline Fatores de riscos ocupacionais* & $f$ & $\%$ & Fatores de riscos ocupacionais* & f & $\%$ \\
\hline Riscos físicos & \multicolumn{2}{|c|}{$(n=97)$} & Muito tempo de pé & 10 & 4.86 \\
\hline Vibrações & 4 & 4.12 & E ncurvado & 5 & 2.43 \\
\hline Ruído & 37 & 38.14 & Na ponta dos pés & 1 & 0.48 \\
\hline Temperatura elevada & 38 & 39.18 & Agachado & 3 & 1.46 \\
\hline Temperatura baixa & 2 & 2.06 & Psicossociais & \multicolumn{2}{|c|}{$(n=238)$} \\
\hline Ventilação precária & 12 & 12.38 & Relaciona-se bem com colegas & 43 & 18.07 \\
\hline Iluminação ina dequada & 2 & 2.06 & Relacionamento ruim com os colegas & 1 & 0.42 \\
\hline Umidade & 2 & 2.06 & Competição com colegas & 1 & 0.42 \\
\hline Químicos & \multicolumn{2}{|c|}{$(n=45)$} & Colaboração com colegas & 2 & 0.84 \\
\hline Gases & 2 & 4.44 & B om relacionamento com chefes no trabalho & 44 & 18.49 \\
\hline Poeira de farinha de trigo & 39 & 86.68 & Relacionamento com chefes & 1 & 0.42 \\
\hline Poeira de aditivo & 4 & 8.88 & Não tem chefe é o empregador & 2 & 0.84 \\
\hline Biológicos & \multicolumn{2}{|c|}{$(n=11)$} & Chega dis pos to no trabalho & 42 & 17.65 \\
\hline Presença de insetos & 1 & 9.09 & Chega indis pos to no trabalho & 3 & 1.26 \\
\hline Presença de roedores & 1 & 9.09 & Condições ao final do trabalho & & \\
\hline Trabalhadores com gripes e res friados & 9 & 81.82 & Fadiga fís ica & 37 & 15.55 \\
\hline Ergonômicos & \multicolumn{2}{|c|}{$(n=206)$} & Fadiga mental & 9 & 3.78 \\
\hline Levantamento e transporte manual de peso & 54 & 26.23 & Tranqüilo & 1 & 0.42 \\
\hline Repetição de movimentos & 41 & 19.90 & Cansado & 1 & 0.42 \\
\hline Ritmo intenso de trabalho & 33 & 16.01 & Não sente cansaço & 4 & 1.68 \\
\hline Uso de máquinas que exigem es forço fís ico & 33 & 16.01 & Perda do sono & 1 & 0.42 \\
\hline Trabalho monótono (parado) & 1 & 0.48 & Realização do trabalho & & \\
\hline Trabalho com as mãos sobre a cabeça ou cotovelos acima dos ombros & 3 & 1.46 & Sozinho & 18 & 7.56 \\
\hline Coluna inclinada & 22 & 10.68 & Em equipe & 28 & 11.76 \\
\hline
\end{tabular}

O n colocado entre parênteses corresponde ao número de respostas dadas pelos sujeitos para cada fator de risco ocupacional.

\section{DISCUSSÃO}

Padeiro é o que planeja a produção e prepara a massa de pão, macarrão e similares; também produz caldas de sorvete, compotas, doces, prepara recheios e confecciona salgados; redige requisições e registros de saída de materiais e relatórios de produção. Trabalha em conformidade as normas e procedimentos técnicos e de qualidade, segurança, higiene, saúde e preservação ambiental (12).

A presença de trabalhadores masculinos e casados coincide com os dados de outro estudo, confirmando a presença de homens na panificação (13). A fabricação de pão é tarefa ancestral masculina e distancia o homem coletor/caçador daquele que cumpre técnicas, transformando alimentos em comida(14); quanto ao nível de escolaridade incompleto pode estar relacionado aos riscos de acidentes, pelo fato dos trabalhadores não se constituírem mão-de-obra especializada (15).

Em relação às características profissionais, a maioria trabalha de 10 a 15 anos na panificação $(34,04 \%)$; caso sejam contabilizados todos os com tempo superior a 10 anos, o percentual eleva-se para 57,45\%. Tal resultado sugere que a maioria dos trabalhadores permanece por um tempo razoável nas atividades, indicando que eles aumentam o número de horas diárias trabalhadas no mesmo serviço ou em outro emprego, visto que este trabalho pode ser desenvolvido em diversos turnos. Percentual considerável (42,55\%) está realizando tal atividade há menos de 10 anos, fato este pode estar relacionado com a não exigência de formação especializada ou a não determinação de nível escolaridade. Quanto ao emprego anterior, 44,66\% não apresentavam experiência constituindo-se o trabalho nas padarias em seu primeiro emprego; anteriormente exerciam atividades variadas e poucas relacionadas à panificação.

Alguns dos RO identificados pelos sujeitos evidenciam a presença de fatores/agentes de riscos no ambiente laboral.

A percepção de risco é difícil de ser com- 
preendida; diversos fatores são conhecidos por influenciá-la e ainda falta uma completa compreensão das maneiras pelas quais as pessoas caracterizam os riscos (9). A compreensão do processo de trabalho na panificação permite o melhor entendimento dos $\mathrm{RO}$ que ocorrem no ambiente de trabalho.

Os RO físicos identificados pelos sujeitos deste estudo foram presença de temperatura elevada $(39,18 \%)$, ruído $(38,14 \%)$ e ventilação precária $(12,38 \%)$. Tais resultados assemelham-se aos de estudo realizado na Romênia em que os autores identificaram nos ambientes de padaria, entre o risco físico, os fatores ruído e temperatura elevada (10). Ruído e o calor foram os agentes físicos mais identificados em indústria da panificação. O ruído é um som indesejável e nocivo à saúde, podendo causar alterações auditivas, circulatórias, digestivas, reprodutoras, além de distúrbios de equilíbrio, do sono, psicológico e social; o calor associado ao tipo de atividade desenvolvida pode ocasionar fadiga, fraqueza, tontura, dor de cabeça, desidratação, câimbras, mal-estar e irritação na pele dos trabalhadores (13). RO físicos para o padeiro foram considerados a exposição do trabalhador a temperaturas extremas (400 a $700^{\circ} \mathrm{C}$ ), em regime de trabalho intermitente; a ventilação natural ou artificial deficiente representa, normalmente, significativas mudanças nas trocas de temperatura ambiente, provocadas pela alteração de abertura e fechamento dos fornos (15).

No que se refere aos fatores de RO químicos, $86,68 \%$ dos sujeitos identificaram a poeira de farinha de trigo como tal. Esse resultado reforça a conclusão de que a exposição profissional ao pó desta farinha é o risco mais comumente detectado na indústria da panificação, conforme já apontados em vários estudos (4-6,8). Os trabalhadores quase sempre estão "brancos" de pó, razão de sofrerem transtornos de saúde $(6,10,15)$. A poeira de farinha de trigo, independente da quantidade e tamanho da partícula pode ser alergênica e causar problemas respirató- rios e dermatológicos, dependendo da suscetibilidade de cada indivíduo $(6,13,15)$. Também foi constatada a presença de poeira dos aditivos químicos utilizados na fabricação de pães $(8,51 \%)$, assim como no estudo britânico (6). Poeiras presentes na indústria de alimentos trazem efeitos para a saúde dos trabalhadores, que podem apresentar dor de garganta e problemas respiratórios, como rinite, asma, bem como lesões nos olhos, por entradas de corpos estranhos (16). A presença de gases nos ambientes de trabalho foi detectada pelos padeiros que utilizam fornos a lenha para assar os pães. Quando chaminés estão obstruídas ou mal projetadas, a combustão da lenha na fornalha pode provocar a acumulação de gases nocivos, incluindo-se o monóxido de carbono, responsável pela ocorrência de asfixia e intoxicações $(10,15)$. Tais dados evidenciam a necessidade de controle ambiental, visando locais de trabalho mais saudáveis, também recomendados no estudo romeno (10).

Quanto aos fatores de riscos ergonômicos relativos ao ambiente de trabalho, 19,9\% dos sujeitos destacaram a repetição dos movimentos na execução do seu trabalho; em seguida o ritmo intenso e o uso de máquinas que exigem esforço físico (16,01\%). Agentes de risco relacionados à ergonomia são considerados aqueles que interferem no equilíbrio entre o trabalho e o homem, podendo provocar danos à saúde do trabalhador por alterações psicofisiológicas, como também comprometer a segurança no ambiente de trabalho e a produtividade (13). Os resultados do presente estudo confirmam esta assertiva: os agentes ergonômicos encontrados assemelham-se, em parte, aos identificados em investigação sobre os trabalhadores de padarias (13); no estudo realizado na Romênia, ficou evidente que os trabalhadores assumiam posição ortostática no trabalho (esforço estático), manipulavam peso (esforço dinâmico) e realizavam movimentos reiterados de apreensão ou extensão da mão e de rotação no antebraço para introduzir o pão no forno 
(10). A organização do trabalho impõe o horário de início da jornada, a postura (posição em pé) e a repetitividade das tarefas, seguidos do levantamento de peso, transporte manual e manuseio da sacaria de farinha de trigo de 50 quilos. Movimentos repetidos são observados na fase de modelação do pão e podem resultar em desordens músculo-esqueléticas; também a posição parada em pé (ortostática) é capaz de colaborar para o aparecimento de varizes $(10,13,15)$.

Posições desconfortáveis junto aos equipamentos para a execução das atividades são assumidas pelos trabalhadores deste estudo. Em relação à posição "coluna inclinada", $10,68 \%$ constantemente a assumem e 4,86\% adotam a posição "muito tempo em pé". O modo da colocação da farinha e a retirada da massa na amassadeira espiral podem exigir postura inadequada e a organização do trabalho na posição ortostática pode causar, além das varizes, o aparecimento de dores lombares (13). Trabalhadores da panificação adotam esforços físicos repetitivos e ritmos intensos, bem como posições desconfortáveis no desempenho de suas atividades de preparo e manipulação da massa de pão (10). Tal situação pode ser estar relacionada a não adequação da posição das máquinas aos trabalhadores, a não alternância de postura, em pé e sentado, ao despreparo quanto à postura correta no transporte e levantamento de peso, a não utilização de carrinho manual para transporte de peso e outros, o que possivelmente causam afecções ósteo-musculares, dentre outros problemas de saúde.

Dos fatores de RO biológicos, $81,82 \%$ dos sujeitos informaram apresentar gripes e resfriados. Estes problemas podem ter sido confundidos com os sintomas como renites, asmas, DPOC, entre outros, descritos em investigações, já que a exposição à poeira da farinha é mencionada como fator de risco para enfermidades respiratórias alérgicas (4$8,16)$. Em menor freqüência foi indicada a presença de insetos e roedores, que contabi- lizam 18,18\% das respostas. Algumas características do ambiente da panificação, como a umidade relativa do ar, temperatura acima de $25^{\circ} \mathrm{C}$ e presença de matéria orgânica, favorecem a proliferação de agentes biológicos e ainda, este risco pode causar alterações de saúde como alergias, verminoses, micoses e infecções do tipo toxoplasmose e leptospirose (13). Há baratas e ratos nas padarias, sobretudo nos locais de armazenamento da lenha utilizada nos fornos, nos sanitários, nos bebedouros, nos filtros e nas lavanderias, quando apresentam precárias condições de asseio e limpeza. No presente estudo constatou-se, nos ambientes de trabalho, a presença de farinha de trigo espalhada ou amontoada no chão a qual, possivelmente, atrai os insetos e roedores mencionados por alguns dos sujeitos; tal situação favorece a permanência destes vetores no ambiente laboral e pode desencadear doenças aos que aspiram a poeira de farinha já contaminada por microrganismos, principalmente durante a varrição do chão. Também se constatou a presença de armadilhas para capturar ratos e baratas nos diversos ambientes, o que confirma a existência destes animais.

Também a falta de higiene corporal dos trabalhadores soma-se aos agentes biológicos presentes no ambiente de trabalho da panificação (15).

Investigação, em Belo Horizonte (Brasil) avaliou as Boas Práticas de Fabricação, que se constituem em um conjunto de normas empregadas em produtos, processos, serviços e edificações, visando a promoção e a certificação da qualidade e da segurança do alimento. Foi realizado em uma indústria de fabricação de pão de queijo. Para o estudo da qualidade microbiológica, foram coletadas amostras das mãos de cada funcionário que participou diretamente no processamento do pão. As mãos, quando higienizados previamente ao processo produtivo, foram amostrados antes e após a higienização. Os resultados mostraram que 59\% apresentaram contagem de 
mesófilas superior a $10^{5} \mathrm{UFC} /$ mão, além da presença de coliformes totais em $93,1 \%$ das amostras, de coliformes fecais em $34,5 \%$ e de Staphylococcus coagulase positiva em $46,7 \%$. Tais resultados indicam hábitos anti-higiênicos por parte dos manipuladores de alimentos, bem como processo de higienização das mãos inadequado, uma vez que $70 \%$ das amostras foram coletadas após a higienização das mesmas (17).

Com relação aos fatores de RO psicossociais, pesquisas têm apontado evidencias sólidas de relação entre eles e a saúde e que fatores de riscos apresentam sua base na organização do trabalho, sendo eles o excesso de demandas ou exigências do trabalho, o escasso controle sobre ele, o baixo apoio social (18), o relacionamento interpessoal, entre outros. Quando indagados sobre o aspecto do relacionamento entre os pares, alguns dos sujeitos (18,07\% e 18,49\% respectivamente) assinalaram manter relacionamento apropriado com os colegas e chefes no trabalho. Trabalhadores da panificação parecem apresentar elevados índices de satisfação profissional, bom relacionamento com os colegas e supervisores no trabalho (13). Quanto às condições físicas e mentais dos sujeitos do estudo no início da jornada laboral, 17,65\% informaram chegar no trabalho "dispostos", enquanto os demais chegam "indispostos" $(1,26 \%)$. Ao final da jornada, a fadiga física $(15,55 \%)$ aparece; tal tipo de fadiga é entendido pela repetição de movimentos, o ritmo intenso de trabalho e o uso de máquinas que exigem esforço físico, além do carregamento de sacarias, elementos que indicam o risco ergonômico. Esta fadiga pode comprometer aqueles que possuem duplo emprego ou que estendem sua jornada diária. Também já foi constatado que a fadiga pode ser ocasionada pelo calor, um dos agentes físicos identificados na indústria de panificação (13).

O trabalho é exercido em equipe (11,76\%), embora algumas vezes também aconteça individualmente $(7,56 \%)$. Este resultado foi confirmado durante a permanência dos autores nos ambientes laborais, constatandose que alguns proprietários contratam entre dois a quatro padeiros, os quais em sua maioria trabalham em duplas; outros mantêm apenas um indivíduo para a realização dos serviços. Tal diferença pode determinar a possibilidade do trabalho em equipe ocorrer com maior freqüência naquelas padarias onde estão contratados dois ou mais padeiros.

Evidencia-se, então, que há a presença de vários agentes de $\mathrm{RO}$ nos ambientes laborais onde acontece o processo da panificação e que os trabalhadores são capazes de reconhecer alguns destes fatores.

\section{CONCLUSÃO}

O estudo permitiu caracterizar o perfil pessoal e profissional dos trabalhadores da panificação de uma cidade brasileira e identificar fatores de RO nos ambientes laborais onde eles trabalham, apesar dos próprios trabalhadores reconhecerem alguns destes agentes de risco.

Os padeiros permanecem por tempo considerável na panificação e esta situação favorece a exposição aos diversos $\mathrm{RO}$, os quais podem geram conseqüências negativas para sua saúde e bem estar. Diante desta realidade, verifica-se que há muitas ações, incluindo-se as de orientação à saúde que podem ser desenvolvidas pela equipe de saúde e de enfermagem, junto a esta população da panificação, visando minimizar ou eliminar sua exposição aos vários fatores de riscos ocupacionais e, principalmente protegê-los dos possíveis agravos à saúde que surgem a partir desses eventos ocupacionais. Empregadores também devem ser alertados para os fatores de $\mathrm{RO}$ e propiciarem ambientes laborais adequados, objetivando a eliminação dos agentes de riscos encontrados. 


\section{REFERÊNCIAS}

1. Vega-Franco L. La salud en el contexto de la nueva salud pública. México: El Manual Moderno/Universidad Nacional Autónoma de México; 2000.

2. Haag GSA, Lopes MJM, Schuck JS. A enfermagem e a saúde dos trabalhadores. 2a ed. Goiânia: AB; 2001.

3. Burgess WA. Identificação de possíveis riscos à saúde do trabalhador nos diversos processos industriais. Belo Horizonte: Ergo; 1997.

4. Jacobs JH, Meijster T, Meijer E, Suarthana E, Heederik D. Wheat allergen exposure and the prevalence of work-related sensitization and allergy in bakery workers. Allergy [Revista em Internet] 2008; 63(12): 1597-604. Hallado em http:// onlinelibrary.wiley.com/doi/10.1111/ j.1398-9995.2008.01698.x/pdf. Acesso em 28 mar. 2011.

5. Baatjies R, Meijster T, Lopata A, Sander I, Rauf-Heimsoth M, Heederik D, et al. Exposure to Flour Dust in South African Supermarket Bakeries: Modeling of Baseline Measurements of an Intervention Study. Ann Occup Hyg [Revista em Internet] 2010; 54(3): 309-18. Hallado em http://annhyg. oxfordjournals.org/content/54/3/309. full.pdf + html. Acesso em 28 mar. 2011.

6. Fraser $\mathrm{S}$, Mason $\mathrm{H}$, Thorpe A, Roberts P, Smith I, Evans G, et al. A study to investigate ways to reduce the dustiness of bakery ingredients and exposure to allergens. United Kingdom: HSE Books; 2010.

7. Rushton L. Occupational causes of chronic obstructive pulmonary disease. Rev Enviro Health [Revista em Internet] 2007; 22(3): 195-212. Hallado em http:// cat.inist.fr/?aModele $=$ afficheN\&cpsi $\mathrm{dt}=$ 19210732. Acesso em 29 mar. 2011.

8. Burdorf A, Lillienberg L, Brisman J. Characterization of exposure to inhalable flour dust in Swedish bakeries. Ann Occup Hyg. 1994; 38(1): 67-78.

9. Alexpoulos EC, Kavadi Z, Bakoyannis G, Papantonopoulos S. Subjective risk assessment and perception in the greek and english bakery industries. J Environ Public Health [Revista em Internet] 2009. Hallado em http://www.hindawi. com/journals/jeph/2009/891754/. Acesso em 29 mar. 2011.

10. Stoia M, Oancea S. Occupational risk assessment in a bakery unit from the District of Sibiu. Acta Universitatis Cibiniensis Series E: FOOD TECHNOLOGY. 2008; 12(2): 11-6.

11. Ministério da Saúde (Br). Conselho Nacional de Saúde. Resolução n. 196 de 10 de outubro de 1996. Normas Regularizadoras de Pesquisa Envolvendo Seres Humanos. Brasília; 1996.

12. Ministério do Trabalho e Emprego (Br). Classificação Brasileira de Ocupações. Brasília: Ministério do Trabalho e Emprego, 2002. Hallado em http://www. mtecbo.gov.br. Acesso em 17 set. 2008.

13. Serviço Social da Indústria. Diretoria de Assistência Médica e Odontológica. Gerência de Segurança e Saúde no Trabalho. Manual de Segurança e Saúde no Trabalho. Coleções Manuais. Indústria da Panificação. São Paulo: Sesi; 2005.

14. Bosisio Júnior A, Lody RGM. O pão na mesa brasileira. 2a ed. Rio de Janeiro: Senac Nacional; 2005.

15. Machado JD, Batista JHL. Levantamento de riscos na indústria da panificação. Recife: Fundacentro; 1982.

16. Sato L, Lacaz FAC. Condições de trabalho e saúde dos trabalhadores do ramo da alimentação. Cadernos de Saúde do Trabalhador. São Paulo: Kingraf; 2000.

17. Tomich RGP, Tomich TR, Amaral CAA, Junqueira RG, Pereira AJG. Metodologia para avaliação das boas práticas de fabricação em indústrias de pão de queijo. Ciênc. Tecnol. Aliment. [Revista em Internet] 2005; 25(1): 115-20. 
Hallado em http://www.scielo.br/scielo. php?script=sci_arttext $\&$ pid $=$ S010120612005000100019\&lng=es\&nrm=iso \&tlng=pt. Acesso em 29 marzo 2011.
18. Benavides FG, Ruiz-Frutos C, García AM. Salud Laboral: Conceptos y técnicas para la prevención de riesgos laborales. 2a ed. Barcelona: Masson; 2000. 